\title{
ON THE EXISTENCE OF STEADY CONVEX SOLUTIONS TO THE REGULARIZED EVOLUTION PROBLEM OF INTERFACES IN THE PLANE
}

\author{
BY \\ M. ROSATI (I.A.C. “M. Picone”, Viale del Policlinico 137, 00161 Roma) \\ AND \\ G. VERGARA CAFFARELLI (Dip. Me. Mo. Mat. Università \\ di Roma "La Sapienza", Via A. Scarpa 10, 00161 Roma)
}

\begin{abstract}
In this paper we study some questions arising in the area of multiphase thermomechanics with interfacial structure. In particular, we prove existence and uniqueness results for steady convex solutions to the regularized problem of interfaces in the plane.
\end{abstract}

1. Introduction. The problem studied in this paper arises in the area of multiphase thermomechanics with interfacial structure (see References [1], [3]). More specifically, the formulation of the regularized problem studied here is found in [2]. The time evolution of the curve $S$ that realizes a convex and unbounded interface may be determined as a function of the angle $\Theta$ of the normal $n$ to $S$ with the $x$ positive semi-axis.

If we denote by $s$ the curvilinear abscissa on $S$, convexity yields invertibility of the mapping $s \rightarrow \Theta(s, t)$ at each time $t$, since the curvature $K(\Theta, t):=\frac{\partial \Theta}{\partial s}$ is never zero. It is proved by Angenent and Gurtin in [1] that the following identity holds:

$$
K_{t}=K^{2}\left(\frac{\partial^{2} V}{\partial \Theta^{2}}+V\right),
$$

where $V$ is the normal velocity of $S$.

When two phases are present, two energies $f(\Theta, t)$ and $F$ are introduced. The first one is the interfacial energy, the second one has constant value and is interpreted as the difference between the bulk energies of the two phases (see [1], [2], [3]). If the energy $f$ is independent of the curvature and a certain kinetic coefficient is constant, the evolution equation derived in [1] has the form

$$
V=g K-F, \quad \text { where } g=f+\frac{\partial^{2} f}{\partial \Theta^{2}} .
$$

The cases $g<0$ and $g>0$ correspond to unstable and stable interfaces respectively.

Received May 31, 1994.

1991 Mathematics Subject Classification. Primary 35Kxx. 
The equation (1.2), together with the identity (1.1), defines an evolution problem of parabolic type, which, in the unstable case $g<0$, is not well posed, since it becomes backward parabolic.

A way to study the unstable case is to assume an interfacial energy $f$ depending on the curvature $K$. In [2], the following constitutive hypothesis is justified:

$$
f(\Theta, t)=f_{0}(\Theta)+\varepsilon K^{2}(\Theta, t)
$$

where $\varepsilon$ is a "small" positive constant. With such an assumption, (1.2) is replaced by the following regularized equation, describing the evolution of a convex interface in the plane:

$$
V=g(\Theta) K(\Theta, t)-\varepsilon K(\Theta, t)\left[\frac{\partial^{2}}{\partial \Theta^{2}} K^{2}(\Theta, t)+K^{2}(\Theta, t)\right]-F,
$$

where $g(\Theta)=f_{0}+d^{2} f_{0} / d \Theta^{2}$ is supposed to be continuous and negative.

REMARK 1. It is useful to recall the following result of [1].

If $K(\Theta)$ is a smooth function defined on the interval $\left[\Theta_{1}, \Theta_{2}\right]\left(0<\Theta_{2}-\Theta_{1}<\pi\right)$, $K(\Theta) \neq 0$ on $\left(\Theta_{1}, \Theta_{2}\right)$ and $K\left(\Theta_{1}\right)=K\left(\Theta_{2}\right)=0$, then $K(\Theta)$ is the curvature of a convex, unbounded boundary curve.

2. The regularized stationary problem. A time-independent solution $(K, V)$ of (1.1), (1.4) is a solution of the following system:

$$
\left\{\begin{array}{l}
K^{2}\left(\frac{d^{2} V}{d \Theta^{2}}+V\right)=0 \\
\varepsilon K\left[\frac{d^{2}(K)^{2}}{d \Theta^{2}}+K^{2}\right]=g K-(F+V)
\end{array}\right.
$$

Recalling Remark 1 in the preceding section and assuming the second equation of (2.1) to be fulfilled at $\Theta_{1}$ and $\Theta_{2}$, we obtain the boundary conditions:

$$
\left\{\begin{array}{l}
K\left(\Theta_{1}\right)=K\left(\Theta_{2}\right)=0 \\
V\left(\Theta_{1}\right)+F=V\left(\Theta_{2}\right)+F=0
\end{array}\right.
$$

Moreover, the system (2.1) can be written:

$$
\left\{\begin{array}{l}
\frac{d^{2} V}{d \Theta^{2}}+V=0 \\
\frac{d^{2}(K)^{2}}{d \Theta^{2}}+K^{2}=\frac{g}{\varepsilon}-\frac{F+V}{\varepsilon K} \quad \text { in }\left(\Theta_{1}, \Theta_{2}\right) .
\end{array}\right.
$$

The Green function of the operator $L(\cdot)=\frac{d^{2}}{d\left(\Theta^{2}\right.}(\cdot)+(\cdot)$ in the interval $\left(\Theta_{1}, \Theta_{2}\right)$ with homogeneous boundary values is given by 


$$
G(\Theta, \xi)= \begin{cases}\frac{\sin \left(\Theta-\Theta_{1}\right) \sin \left(\xi-\Theta_{2}\right)}{\sin \left(\Theta_{2}-\Theta_{1}\right)}, & \Theta_{1}<\Theta \leq \xi<\Theta_{2}, \\ \frac{\sin \left(\xi-\Theta_{1}\right) \sin \left(\Theta-\Theta_{2}\right)}{\sin \left(\Theta_{2}-\Theta_{1}\right)}, & \Theta_{1}<\xi \leq \Theta<\Theta_{2} .\end{cases}
$$

It follows from $(2.4)$ that $G(\Theta, \xi)<0$ in $\left(\Theta_{1}, \Theta_{2}\right) \times\left(\Theta_{1}, \Theta_{2}\right)$, whereas $G\left(\Theta_{1}, \xi\right)=$ $G\left(\Theta_{2}, \xi\right)=0$ (recall that $0<\Theta_{2}-\Theta_{1}<\pi$ ).

We take $F \neq 0$ (see Remark 3 below).

From (2.2), (2.3) we have

$$
V+F=F \int_{\Theta_{1}}^{\Theta_{2}} G(\Theta, \xi) d \xi
$$

Thus,

$$
(F+V) F<0 \quad \text { in }\left(\Theta_{1}, \Theta_{2}\right)
$$

So we are led to look for solutions of $(2.3)$ in the nonnegative unknown $K^{2}$ that satisfies the inequality

$$
(F+V) K>0 \quad \text { in }\left(\Theta_{1}, \Theta_{2}\right)
$$

The last two inequalities imply that on the same interval we have

$$
V F<0, \quad F K<0 .
$$

We will therefore look for solutions of $(2.2),(2.3)$ satisfying the inequality

$$
K V>0 \text { in }\left(\Theta_{1}, \Theta_{2}\right)
$$

We note that, since (2.5) and (2.8) imply that $(F+V) V>0$, we also see that (2.9) implies (2.7).

REMARK 2. With the terminology of [1], condition (2.9) corresponds to a "receding bump".

If we set $\varepsilon=0$ in (1.3), the stationary, nonregularized problem becomes

$$
\left\{\begin{array}{l}
\frac{d^{2} V}{d \Theta^{2}}+V=0 \\
g K-(F+V)=0
\end{array}\right.
$$

In the case of an unstable interface energy $(g<0)$, any solution of $(2.10)$ satisfies, besides (2.6), the inequality $K(F+V)<0$, and therefore also $K V<0$. It follows that no solution of (2.10) may be obtained as a limit, for $\varepsilon \rightarrow 0^{+}$, of solutions of $(2.2),(2.3),(2.9)$. This agrees with a result established in [1], concerning the nonexistence of "receding bumps" for globally unstable energies. 


\section{Solution of the stationary regularized system.}

THEOREM 1 (Existence and uniqueness of the receding bump). Let $g(\Theta)<0$. Then, problem (2.2), (2.3) has one and only one solution $(K, V)$ such that $K V>0$ in $\left(\Theta_{1}, \Theta_{2}\right)$.

Proof. In view of what has been observed in the preceding section, it is enough to show that (2.2), (2.3) has one and only one solution satisfying the inequality (2.7).

Suppose, for example, that $F<0$. Choose a smooth $K_{0}(\Theta)>0$ and define

$$
H_{0}(\Theta)=\int_{\Theta_{1}}^{\Theta_{2}} G(\Theta, \xi)\left[\frac{g(\xi)}{\varepsilon}-\frac{V(\xi)+F}{\varepsilon K_{0}(\xi)}\right] d \xi>0 \quad \text { in }\left(\Theta_{1}, \Theta_{2}\right)
$$

Consider now the following iterative scheme:

$$
\left\{\begin{array}{l}
K_{i}=\sqrt{H_{i-1}} \\
H_{i}=\int_{\Theta_{1}}^{\Theta_{2}} G(\Theta, \xi) R_{i}(\xi) d \xi, \quad i=1,2, \ldots
\end{array}\right.
$$

where

$$
R_{i}(\xi)=\frac{g(\xi)}{\varepsilon}-\frac{V(\xi)+F}{\varepsilon\left(\int_{\Theta_{1}}^{\Theta_{2}} G(\xi, \tau)\left[\frac{g(\tau)}{\varepsilon}-\frac{V(\tau)+F}{\varepsilon K_{i}(\tau)}\right] d \tau\right)^{1 / 2}} .
$$

One proves, by induction, that $H_{i}>0$ in $\left(\Theta_{1}, \Theta_{2}\right)$; moreover,

$$
\left|R_{i}(\xi)\right| \leq\left|\frac{g(\xi)}{\varepsilon}\right|+\frac{|V(\xi)+F|}{\varepsilon\left(\int_{\Theta_{1}}^{\Theta_{2}} G(\xi, \tau) \frac{g(\tau)}{\varepsilon} d \tau\right)^{1 / 2}} .
$$

Therefore, the sequence $\left\{H_{i}(\Theta)\right\}$ is equibounded; in addition, differentiating the second equation of (3.2) with respect to $\Theta$ and using (3.4), one sees that it is also equicontinuous. The proof that a solution having the requested properties exists is completed by passing to the limit for a uniformly convergent subsequence.

To prove uniqueness, we begin by observing that, if we write $U=K^{2}$, the condition $(F+V) K>0$ allows us to write the second equation (2.3) as

$$
\left\{\begin{array}{l}
\frac{d^{2} U}{d \Theta^{2}}+U=\frac{g}{\varepsilon}-\frac{|V+F|}{\varepsilon \sqrt{U}} \\
U\left(\Theta_{1}\right)=U\left(\Theta_{2}\right)=0
\end{array}\right.
$$

We proceed by contradiction. Let

$$
\alpha=\frac{\pi}{\Theta_{2}-\Theta_{1}}>1
$$

Since the eigenvalues $\lambda$ of the equation $U_{\infty}+\lambda U=0$ with homogeneous boundary values are $\lambda=n^{2} \alpha^{2}, n=1,2, \ldots$, each smooth function $\Phi$ that vanishes at the boundary points $\Theta_{1}, \Theta_{2}$ satisfies the inequality

$$
\int_{\Theta_{1}}^{\Theta_{2}}\left(\Phi_{\Theta}\right)^{2} d \Theta \geq \alpha^{2} \int_{\Theta_{1}}^{\Theta_{2}}(\Phi)^{2} d \Theta
$$

Let $U_{1}, U_{2}$ be two solutions of (3.5). We have

$$
\left(U_{2}-U_{1}\right)_{\infty}+\left(U_{2}-U_{1}\right)=\frac{|F+V|\left(U_{2}-U_{1}\right)}{\varepsilon \sqrt{U_{2}} \sqrt{U_{1}}\left(\sqrt{U_{2}}+\sqrt{U_{1}}\right)} .
$$


Multiplying this equation by $\left(U_{2}-U_{1}\right)$ and integrating on $\left(\Theta_{1}, \Theta_{2}\right)$, we obtain

$$
-\int_{\Theta_{1}}^{\Theta_{2}}\left[\left(U_{2}-U_{1}\right)_{\Theta}\right]^{2} d \Theta+\int_{\Theta_{1}}^{\Theta_{2}}\left(U_{2}-U_{1}\right)^{2} d \Theta=\int_{\Theta_{1}}^{\Theta_{2}} \frac{|F+V|\left(U_{2}-U_{1}\right)^{2}}{\varepsilon \sqrt{U_{2}} \sqrt{U_{1}}\left(\sqrt{U_{1}}+\sqrt{U_{2}}\right)} d \Theta>0 .
$$

Therefore, in view also of (3.7), we have

$$
\left(1-\alpha^{2}\right) \int_{\Theta_{1}}^{\Theta_{2}}\left(U_{2}-U_{1}\right)^{2} d \Theta>0 .
$$

The inequalities (3.8) and (3.6) are incompatible.

REMARK 3. If $F=0$, then $V \equiv 0$ and the problem reduces to $L(H)=\frac{g(\Theta)}{\varepsilon}<0$ with homogeneous boundary conditions. In this case, there are two static solutions:

$$
K= \pm \sqrt{\frac{1}{\varepsilon} \int_{\Theta_{1}}^{\Theta_{2}} G(\Theta, \xi) g(\xi) d \xi}
$$

TheOREM 2 (Existence of "advancing bumps" for "small" values of $\varepsilon$ ). Let $0<m=$ $\min \{-g(\Theta)\}, \Theta \in\left[\Theta_{1}, \Theta_{2}\right]$, and $\frac{1}{\varepsilon} \geq \frac{8}{m^{3}} F^{2}\left|\int_{\Theta_{1}}^{\Theta_{2}} G(\Theta, \xi) d \xi\right|$. Then, the system $(2.2),(2.3)$ has a solution $(K, V)$ such that $K V<0$ in $\left(\Theta_{1}, \Theta_{2}\right)$.

Proof. Again we assume, for simplicity, that $F<0$; and thus, $F+V>0$ since the function $F+V$ satisfies Eq. (2.5). For

$$
H_{0}=-a \int_{\Theta_{1}}^{\Theta_{2}} G(\Theta, \xi) d \xi, \quad \text { where } a=\frac{m}{2 \varepsilon}
$$

we consider the iterative scheme

$$
H_{i}=\int_{\Theta_{1}}^{\Theta_{2}} G(\Theta, \xi) R_{i}(\xi) d \xi
$$

where

$$
R_{i}(\xi)=\frac{g(\xi)}{\varepsilon}+\frac{V(\xi)+F}{\varepsilon\left(\int_{\Theta_{1}}^{\Theta_{2}} G(\xi, \tau)\left[\frac{g(\tau)}{\varepsilon}+\frac{V(\tau)+F}{\varepsilon \sqrt{H_{i-1}(\tau)}}\right] d \tau\right)^{1 / 2}}
$$

We want to show that

$$
R_{i}(\xi) \leq-a, \quad i=1,2, \ldots
$$

Note the following identities:

$$
R_{1}(\xi)=\frac{g(\xi)}{\varepsilon}+\frac{V(\xi)+F}{\varepsilon \sqrt{H_{0}(\xi)}} ; \quad \frac{V(\Theta)+F}{\sqrt{H_{0}(\Theta)}}=\frac{|F|}{\sqrt{a}}\left|\int_{\Theta_{1}}^{\Theta_{2}} G(\Theta, \xi) d \xi\right|^{1 / 2} .
$$

Note also that, from the definition of $a$ and the assumption on $\varepsilon$, it follows that

$$
\sqrt{a}(-\varepsilon a-g) \geq \frac{m}{2} \sqrt{a} \geq|F| \sqrt{\left|\int_{\Theta_{1}}^{\Theta_{2}} G(\Theta, \xi) d \xi\right|}
$$


and thus

$$
-a \geq \frac{g(\xi)}{\varepsilon}+\frac{V(\xi)+F}{\varepsilon \sqrt{H_{0}(\xi)}}=R_{1}(\xi) .
$$

This implies that $\sqrt{H_{1}} \geq \sqrt{H_{0}} \geq \frac{V+F}{-\varepsilon a-g}$, which establishes (3.11).

Furthermore, it is easy to check that $R_{i}(\xi) \geq \frac{g(\xi)}{\varepsilon}$. From this inequality and (3.11) it follows that a subsequence of $\left\{H_{i}\right\}$ converges to a function $H \geq 0$. By taking $K=$ $-\sqrt{H} \leq 0$, we can construct an advancing bump.

\section{REFERENCES}

[1] S. Angenent and M. E. Gurtin, Multiphase thermomechanics with interfacial structures. 2 Evolution of an isothermal interface, Arch. Rational Mech. Anal. 108, 323-391 (1989)

[2] A. DiCarlo, M. E. Gurtin, and P. Podio-Guidugli, A regularized equation for anisotropic motionby-curvature, Siam J. Appl. Math. 52, 1111-1119 (1992)

[3] M. E. Gurtin, Thermomechanics of Evolving Phase Boundaries in the Plane, Oxford Science Publications, Clarendon Press, Oxford, 1993

[4] M. H. Protter and H. F. Weinberger, Maximum Principle in Differential Equations, Prentice-Hall, Inc., 1967 